\title{
Novel Gait for an Anthropoid and Its Joint Demeanors while Stepping Up and Down Stairs
}

\author{
Md. Akhtaruzzaman", Amir A. Shafie \\ Department of Mechatronics Engineering, Kulliyyah of Engineering, International Islamic University Malaysia, \\ Kuala Lumpur, 53100, Malaysia
}

\begin{abstract}
A biped humanoid is considered as an open kinematic chain consisting of two sub chains as legs and torso which connected at a common point, the hip. When defining the gait for stepping stairs, there exist two main phases which are Single Support Phase (SSP) and Double Support Phase (DSP). The challenge is to make the anthropoid stable in its upright position without losing it while performing the two alternating phases, SSP and DSP. In this paper a novel gait for an anthropomorphic system to step up-down stairs is formulated and experimented using BIOLOID humanoid platform. The gait is merged with various poses which are excogitated through the Forward and Inverse Kinematics (FK and IK) analysis based on Denavit-Hartenberg (D-H) representation and Geometric-Trigonometric (G-T) formulation techniques. The main focus of this paper is to represent and analyze the diverse behaviour of the various joint actuators of the robot while performing the step up and down stairs.
\end{abstract}

Keywords Humanoid Robot, Stairs Climbing Gait, Step Up Down Stairs, Gait Analysis

\section{Introduction}

Gait for moving forward or backward or even turning is defined as the alternating phases of single and double support mode of an anthropomorphic robot. The DSP is the phase when both legs are in contact with the navigation surface. In SSP only a single leg is in contact with the ground which is called as the stance leg while the other one is called as the swing leg. The main requirement of the robot is to alternate the phases by maintaining the displacement of the horizontal components such as the Centre of Mass (CoM), with strictly monotonic fashion which means the CoM should be stable without rocking forward and backward. In order to understand the gait, it is necessary to visualize the human planes of sections. The Sagittal Plane divides the body into right and left sections. The Frontal or Coronal Plane separates the body into front and back portions. Finally the Transverse Plane is perpendicular to both the Sagittal and Frontal Planes and divides the body up and down portions at the hip position of the body [1,2]. These three different planes are represented in Figure 1.

Leonardo de Vinci is considered as the first man who has drawn a humanoid mechanism in 1495 [3,4]. Construction and development period of humanoid system commences in the $19^{\text {th }}$ century when John Brainerd invented the Steam Man

* Corresponding author:

akhter900@yahoo.com (Md. Akhtaruzzaman)

Published online at http://journal.sapub.org/jmea

Copyright (C) 2011 Scientific \& Academic Publishing. All Rights Reserved in $1865[4,8]$. At the beginning of $20^{\text {th }}$ century an evolutionary number of humanoid systems appear such as ELEKTRO, BIPER, Tron-Xm, H6, Waseda Legged series, WABOT, WABIAN family, WAP series, SAIKO, E0 to E6, P1 to P3 and so on [4]. Jumping robot, Spring Turkey, Spring Flamingo, Uniroo, Biped Planar, and 3D Biped were the remarkable invention from MIT having noteworthy performance on walking and running movements with dynamic and stable gait. ASIMO the currently most advance humanoid system is demonstrated in the year $2000[5,6,9]$. BIP2000, AIBO, RABBIT, KHR, HUBO, HRP, HOAP, ROBIAN, NAO, iCub, CB2, MAHRU, REEM, QRIO are some of the greatest anthropoid platform came out during the last ten years [4-9].

A type of walking pattern and sensory feedback control strategy were proposed for an anthropomorphic robot stepping up stairs, in 2008 [10]. The parameter selection for the designed gait was excogitated based on the Constrained Nonlinear Optimization Problem (CNOP). Using the Reinforcement Learning (RL) method the control parameters for feedback were adjusted for stepping up the stairs. An algorithm was proposed by S. H. Kim and Y. Sankai to generate extended task for anthropoid to perform various movements including the ascending steps [11]. The third-order Bezier curve was adopted for this experiment where the desired trajectory was possible to accomplish by modifying the control parameters. Accelerometer and Force sensor based control mechanism for a humanoid system stepping up stairs was explicated by T. H. S. Li et al. [12] where an autonomous control strategy was proposed. Self balancing gait using Fuzzy Logic (FL) and Zero Moment Point (ZMP) were 
adopted for this experiment and ten step stairs climbing strategy was demonstrated with successful accomplishment. Robust model based real time 3D tracking strategy was demonstrated in 2007 where a programmable graphics hardware was used to operate the humanoid robot [13].

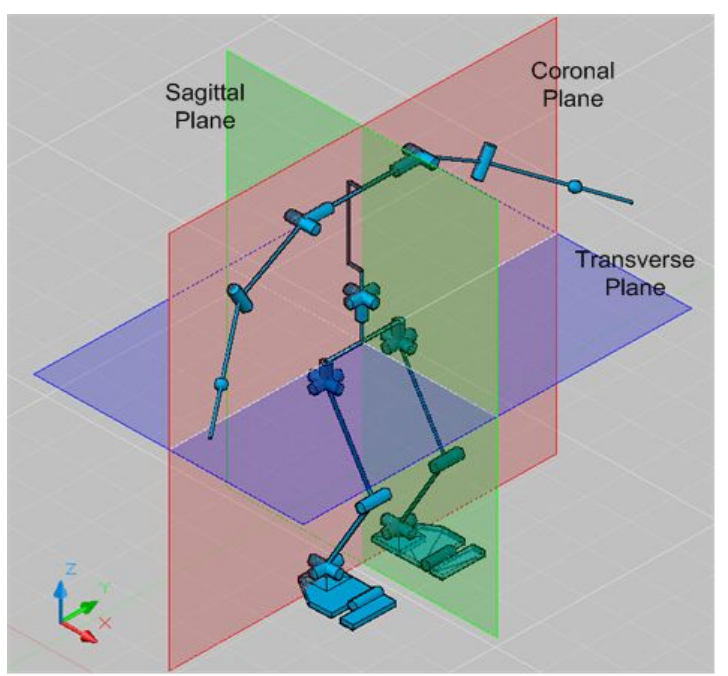

Figure 1. Three different Section Planes of an anthropomorphic system

All the methods, mentioned above, have demonstrated with the robustness of their individual gaits having the same type of foot placing strategy as, Flat-Lifting and Flat-Landing (FL-FL) footsteps. This technique is feasible for slow walking patterns. In this paper a novel gait is designed and demonstrated using the BIOLOID humanoid platform where human like walking is demonstrated and can be abridged as, Heel-Contact and Toe-Off (HC-TO) walking strategy. This particular method may provide the ability to the anthropomorphic system for faster walking and even for running. The paper also represents the CoP-CoM tracking strategy which is excogitated by following the ZMP technique and can be defined as, tracking the Centre of Pressure (CoP) movements by CoM of the system. The paper mainly focuses on the gait design strategy and various movement behaviors of the joint actuators in performing the gait for step up-down stairs.

Pre formulation of gait vector and designing the gaits for Ascending-Descending stairs are presented in Section 2. In Section 3, the various angular positions of the joint actuators are tabulated. The deportments of the joint actuators are also plotted and analyzed in the same section. Finally, Section 4 presents the abridgement of the paper.

\section{Gait Analysis and Design}

\subsection{Analysis and Pre Formulation}

Humanoid gaits are the combination of various postures and poses. Some series of poses can be accumulated together as a page and a group of pages are combined with an adjustable sequence to make the robot able to achieve the next stage of the performing gait. If the system begins to execute a particular action, the execution of a particular page should be completed before the start of the next action. A mathematical expression for the execution process can be defined with $\vec{P}$, which is considered as a vector and a function of the joint vector $\vec{Q}$. The $\vec{P}$ indicates the pose of the humanoid for a certain moment of time in a particular direction while executing a page. If the start and end poses of a single page is indicated as $P_{i}$ and $P_{f}$, a general equation can be formulated as shown in Equation (1) and Equation (2) where $\vec{Q}_{(i)}$ is a set of the joint vectors of ' $i$ 'th pose contingent on the ' $n$ ' DoF of the system.

$$
\begin{gathered}
\vec{P}=\overrightarrow{P_{i} P_{f}}=\sum_{i}^{f} f\left(\vec{Q}_{(i)}\right) \\
\vec{Q}_{(i)}=\left\{\vec{Q}_{1(i)}, \vec{Q}_{2(i)}, \vec{Q}_{3(i)} \ldots \ldots \ldots, \vec{Q}_{n(i)}\right\}
\end{gathered}
$$

\subsection{Defining the Stair Constraints}

Moving up and down stairs are the complex task for a humanoid where more stable gaits are required. During the climbing stairs, the humanoid has to move its CoM position upwards to shift its torso on the next step which is more complex than moving the CoM while navigating on flat surface. Step down stairs is also the difficult and complex chore to remain stable while gravitational force attracts the $\mathrm{CoM}$ point. Figure 2 represents the designed stairs having two steps up and down. Height of the each step is about 3.1 $\mathrm{cm}$ which makes $6.2 \mathrm{~cm}$ height in total for the two steps. The stairs are especially designed for the humanoid with the necessary space to place its feet on the steps.

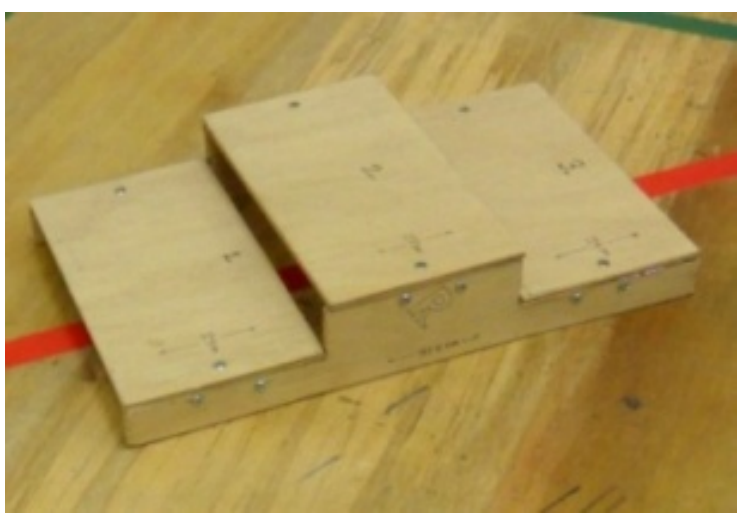

Figure 2. Two step stairs model

\subsection{Gait for Ascending Stairs}

As the two swing modes are involved to gait the humanoid for a single step up stairs, the designed gait is conceived with fifteen poses. The first three poses, shown in Figure 3(a) and (b) are considered as same as the walking or obstacle overcoming gait design. The first swing phase for the action leg has to execute five poses to settle the action foot on the step of the stairs as shown in Figure 3(b) to (f). During this action the CoM of the system is shifted backwards for a little distance to make the humanoid stable without falling down, Figure 3(e). With the proper placement of the front foot on the step, the humanoid moves its torso forward in two different poses, Figure $3(\mathrm{~g})$ and $(\mathrm{h})$. Before the beginning of the 
second swing phase with the rear foot, the anthropoid moves its CoM gradually upwards to fix its stability by front foot support, Figure 3 (i). The second swing phase continues with the next four poses as shown in Figure 3 (j) to (m) and finally the robot moves its torso upwards to come to the Action Pose again. The whole process can be performed in two ways depending on the action leg, right or left. As a result, the algorithm is designed with two functions, StUP_Left() and StUP_Right (). The mathematical description of the procedure can be presented as follows where $\vec{P}_{S U}$ stands for Step Up vector.

$$
\begin{gathered}
\vec{P}_{S U}=\overrightarrow{P_{i} P_{f}}=\sum_{i=1}^{f=15} f\left(\vec{Q}_{(i)}\right) \\
=\sum_{i=1}^{f=15} f\left(\vec{Q}_{a(i)}, \vec{Q}_{b(i)}, \vec{Q}_{c(i)}, \vec{Q}_{d(i)}, \vec{Q}_{e(i)}\right)
\end{gathered}
$$

Here $\vec{Q}_{(i)}$ stands for a set of the joint vectors in a certain pose of the system. Figure 3 presents the design of the fifteen poses to perform step up stairs.

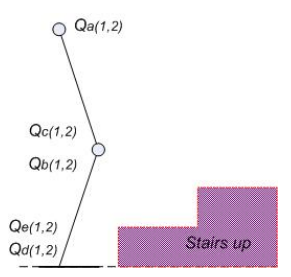

(a)

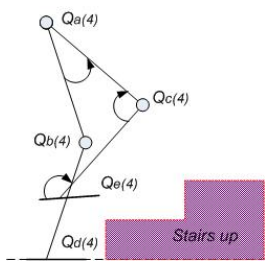

(c)

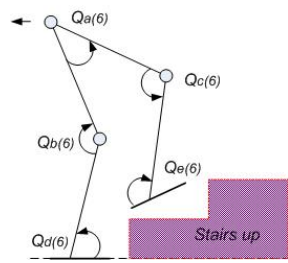

(e)

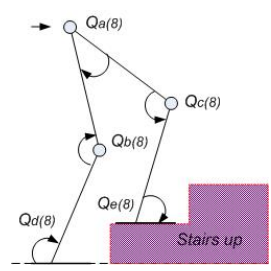

(g)

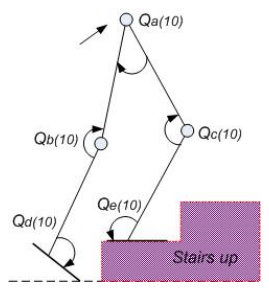

(i)

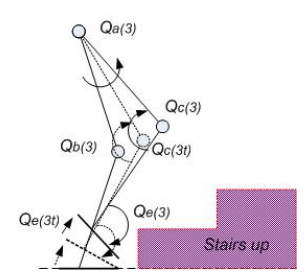

(b)

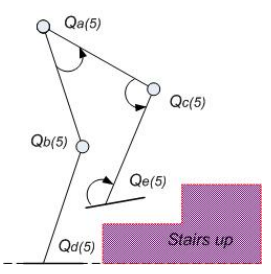

(d)

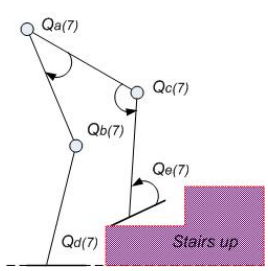

(f)

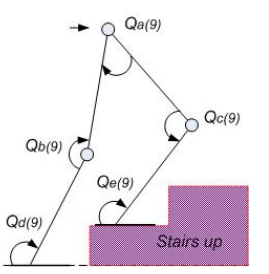

(h)

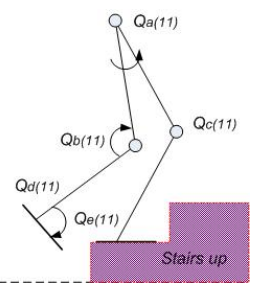

(j)

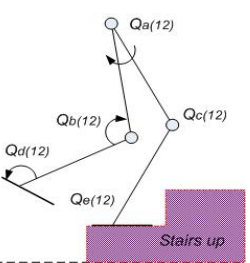

(k)

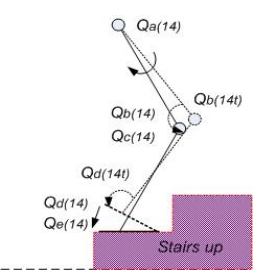

(m)

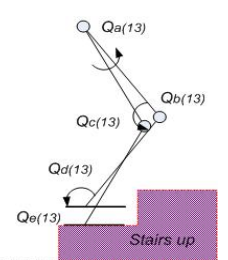

(1)

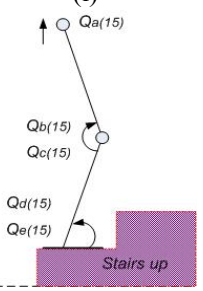

(n)
Figure 3. Fifteen different poses for a single step up of the stairs, (a) Action Pose and Tilt $\mathrm{a}_{\mathrm{a}}$ Pose (two poses), (b) DS-SS $\mathrm{a}_{\mathrm{a}}$ Pose, (c) Foot ${ }_{\mathrm{a}}$ Lifting Pose, (d) Foot ${ }_{\mathrm{a}}$ Forward Pose, (e) Foot ${ }_{\mathrm{a}}$ Adjust Pose, (f) SS-DS a Pose, (g) SS-DS $_{\mathrm{a}}$ Complete Pose, (h) Tilt $\mathrm{b}_{\mathrm{b}}$ Pose, (i) CoM Lifting Pose, (j) DS-SS Pose, (k) Foot $t_{\mathrm{b}}$ Lifting Pose, (1) Foot ${ }_{\mathrm{b}}$ Forward Pose, (m) SS-DS $\mathrm{b}$ Pose and (n) Action Pose

The StUP_Left () and StUP_Right () functions are defined where the series of poses are executed sequentially. To define the main function for the Stair Up Step, StUP () is defined, Figure 4. The function takes a parameter value indicating the left or right leg in action to perform the task. The return value, 0 , represents the system as in Action Pose and the completion of the operation successfully.

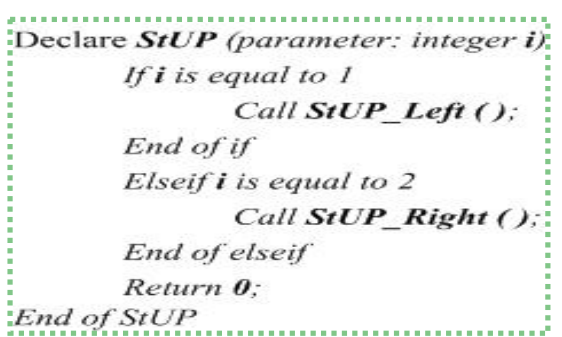

Figure 4. Defining the Step Up function as $\operatorname{StUP}$ () with a parameter value indicating the action leg

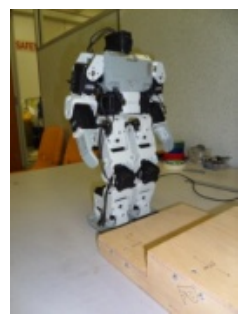

(a)

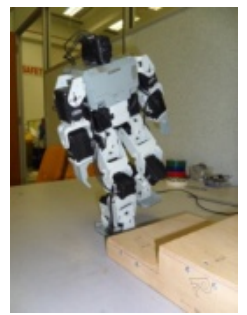

(d)

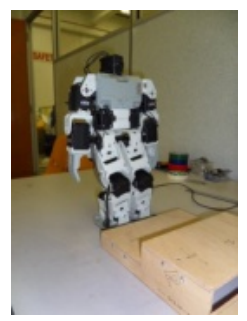

(b)

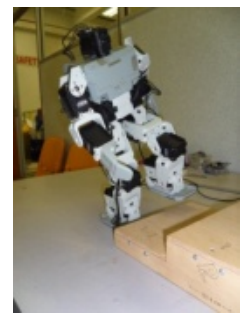

(e)

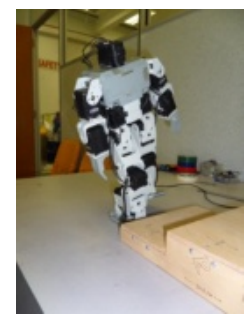

(c)

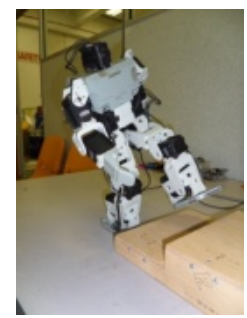

(f) 


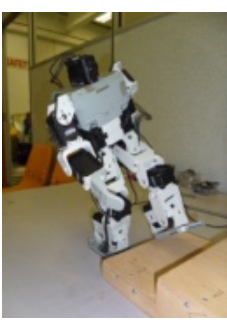

(g)

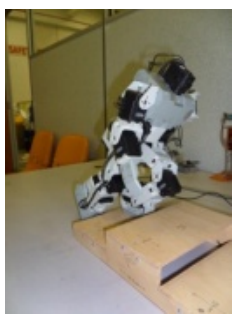

(j)

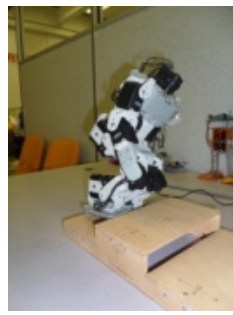

(m)

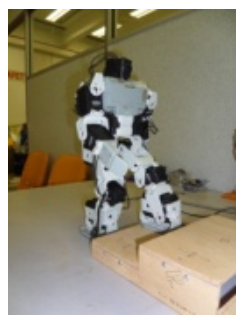

(h)

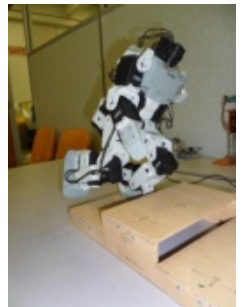

(k)

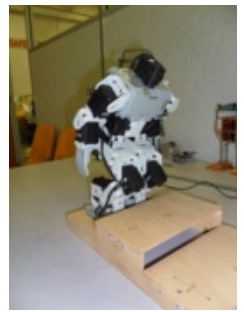

(n)

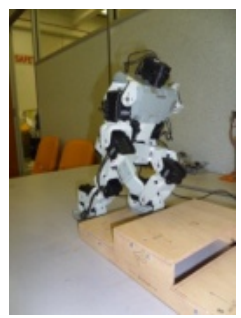

(i)

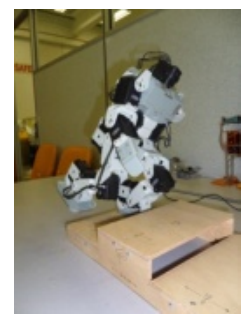

(I)

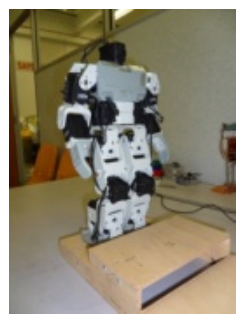

(o)
Figure 5. Various poses for step up stairs experimented using BIOLOID humanoid

\subsection{Fashion of Descending Stairs}

Gait for Step down stairs is one of the critical tasks for the humanoid where total of seventeen poses are executed sequentially. After the Action Pose of this gait, the humanoid drops its CoM point downwards by bending both of the knees as shown in Figure 6 (b). After that the robot tilts to one side to start the first swing phase. The swing phase continues with three poses and ends up by touching the down step with the heel of the swing foot as shown in Figure 6 (c) to $(\mathrm{g})$. During the next four poses the system moves its torso forward, upward, forward and finally a little bit backward to ensure the stability transfer from rear leg to front leg. The second swing phase starts after this action as shown in Figure $6(\mathrm{k})$. Next five poses are performed one after another to reach at the final stage of the second swing phase, Figure 6 (p). The whole process is completed with the Action Pose of the bipedal system. Based on the general equation as represented in Equation (1) and Equation (2), the strategy is formulated as,

$$
\begin{gathered}
\vec{P}_{S D}=\overrightarrow{P_{i} P_{f}}=\sum_{i=1}^{f=17} f\left(\vec{Q}_{(i)}\right) \\
=\sum_{i=1}^{f=17} f\left(\vec{Q}_{a(i)}, \vec{Q}_{b(i)}, \vec{Q}_{c(i)}, \vec{Q}_{d(i)}, \vec{Q}_{e(i)}\right)
\end{gathered}
$$

Here $\vec{P}_{S D}$ represents the Step Down Stairs vector as the function of a set, $\vec{Q}_{(i)}$, of the joint vectors in a certain pose of the gait. The gait may start by activating any of the left or right leg. The functions, StDN_Left () and StDN_Right () is

possible to define where the series of poses are executed by the system in a serial manner. Defining the main function for Step Down Stairs as $S t D N$ (), as presented in Figure 7.

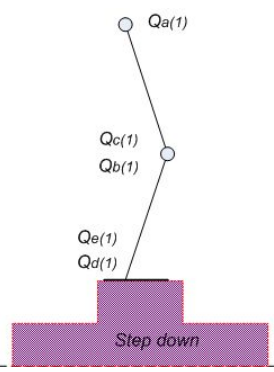

(a)

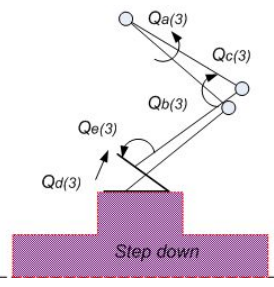

(c)

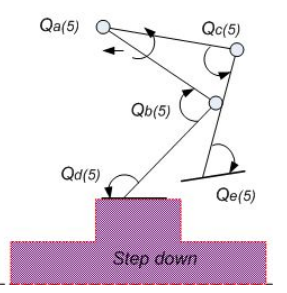

(e)

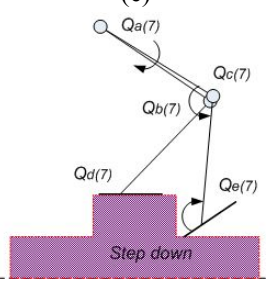

(g)

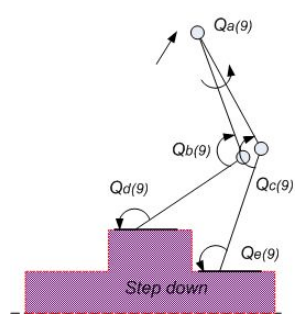

(i)

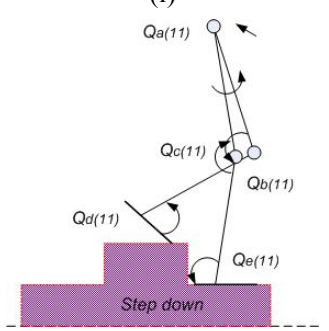

(k)

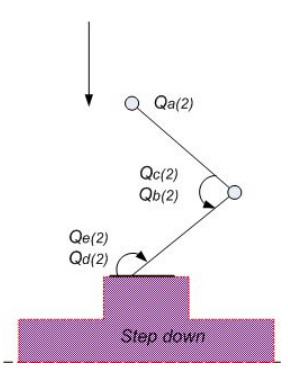

(b)

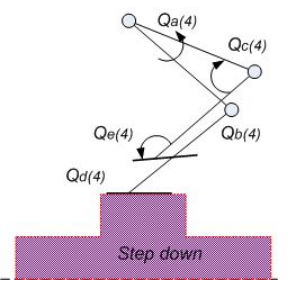

(d)

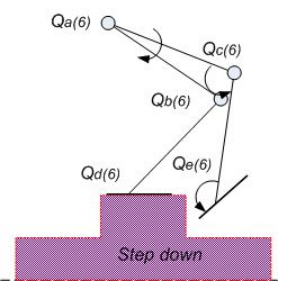

(f)

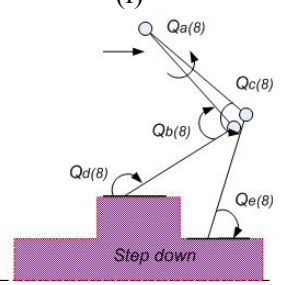

(h)

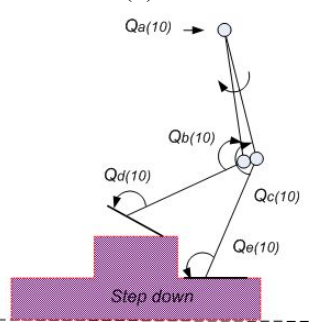

(j)

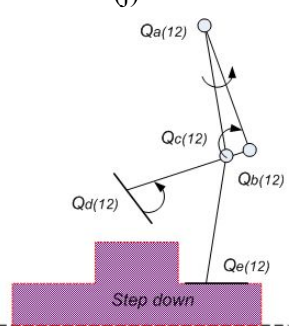

(1) 


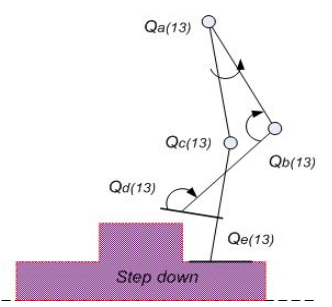

(m)

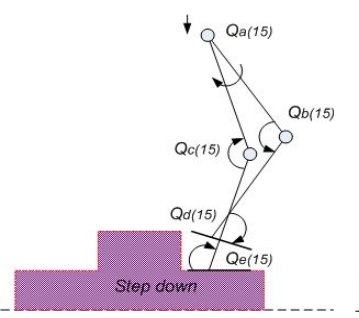

(o)

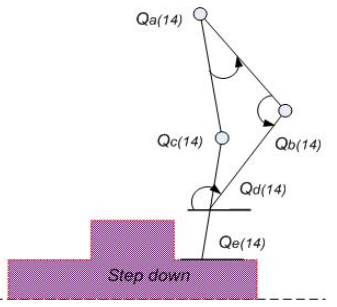

(n)

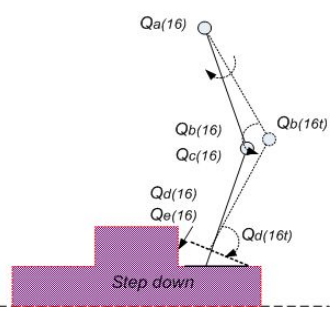

(p)

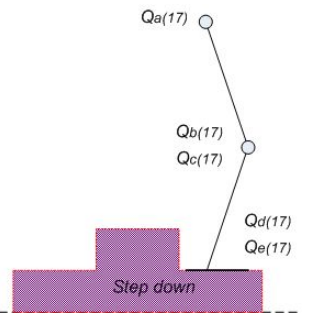

(q)

Figure 6. Seventeen various poses to perform a single step down of the stairs, (a) Action Pose, (b) CoM Drop Pose, (c) Tilt ${ }_{\mathrm{a}}$ Pose, (d) DS-SS a $_{\mathrm{a}}$ Pose (e) Foot Forward Pose, (f) Foot $_{\mathrm{a}}$ Adjust Pose, (g) SS-DS Pose, (h) SS-DS $_{\mathrm{a}}$ Complete Pose, (i) CoM Lifting Pose, (j) CoM Forward Pose, (k) Tilt $\mathrm{b}_{\mathrm{b}}$ Pose

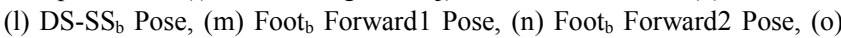
Foot $_{\mathrm{b}}$ Adjust Pose (p) SS-DS Pose and (q) Action Pose

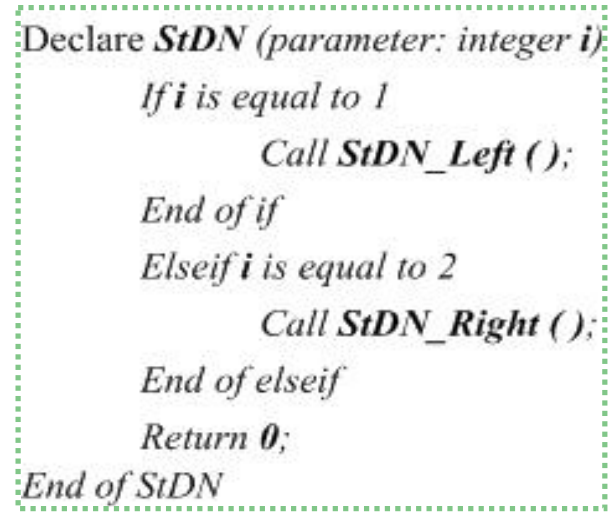

Figure 7. Defining the Step Down function as $S t D N$ () with a parameter value indicating the action leg

Figure 8, bellow, illustrates the various poses for step down stairs applied on the BIOLOID humanoid system.

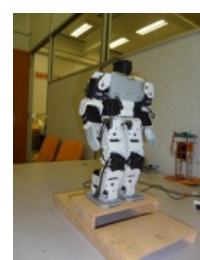

(a)

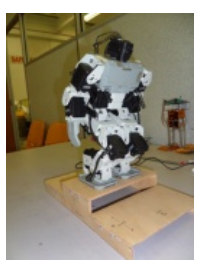

(b)

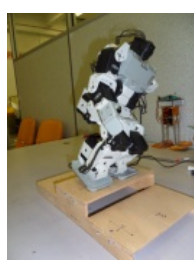

(c)

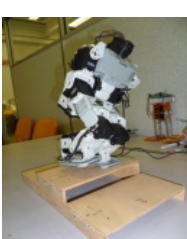

(d)

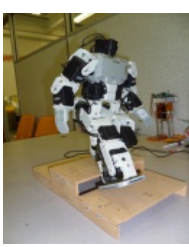

(g)

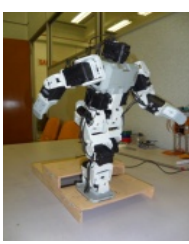

(j)

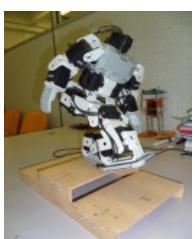

(e)

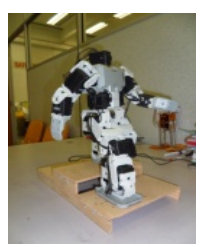

(h)

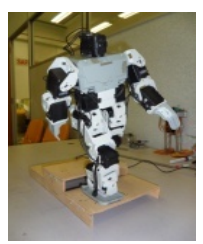

(k)

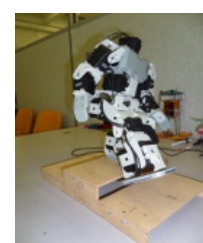

(f)

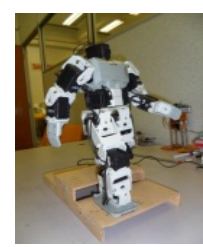

(i)

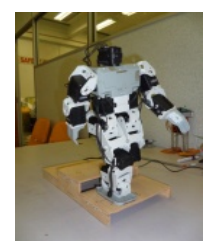

(1)

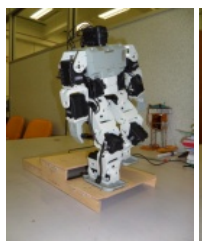

(m)

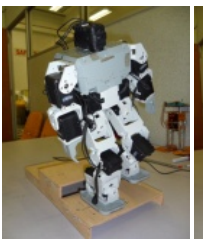

(n)

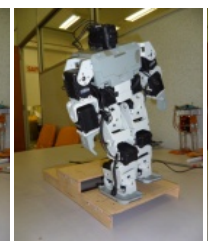

(o)

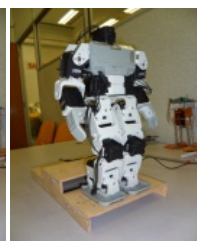

(p)
Figure 8. Various poses for step down stairs applied on BIOLOID humanoid where (p) represents the SS-DS $\&$ Action poses

\section{Joint Demeanors and Discussions}

The various angular positions of the rotary joint actuators are represented in a tabulated format. All the required angular positions are calculated depending on the various poses of the various gaits where the Geometrical Analysis Technique (GAT) is imposed. The findings of the GAT strategy are experimented on the real time system where a small-scale adjustment is enforced to establish the robustness of the various gaits for the robotic platform. These adjustments are necessary because of the backlash errors of the servo actuators gear heads. Finally the table which is required for each gaits are updated, as shown in Figure 9, and fixed into the robot controller.

To observe the behaviors of the various actuators of the system, the tabulated pose angles of the different gaits are simulated in MatLab using the General Spline Interpolation method as Spline $=\operatorname{spapi}(k, x, y)$ and fnplt(Spline ), where the first argument in spapi( ), $k$, is the order of the interpolating Spline.

\subsection{Analysis of Joint Behaviors}

Ascending and Descending stairs are the laborious task for 
the biped system. In this section, the two separate gaits with different behavior of joint movement patterns are observed for the Up going and the Down going steps. The mirror joint actuator movement characteristics of the performed actions are compared by simulating the tabulated values of the designed gait patterns of the humanoid platform. Figure 10 combines the various trajectories for the Shoulder and Elbow joint actuators where the robot performs the one step up and one step down of the stairs. The rest of the figures from Figure 11 to Figure 16 express the various deflections that occur on the lower torso actuators for the same experiment.

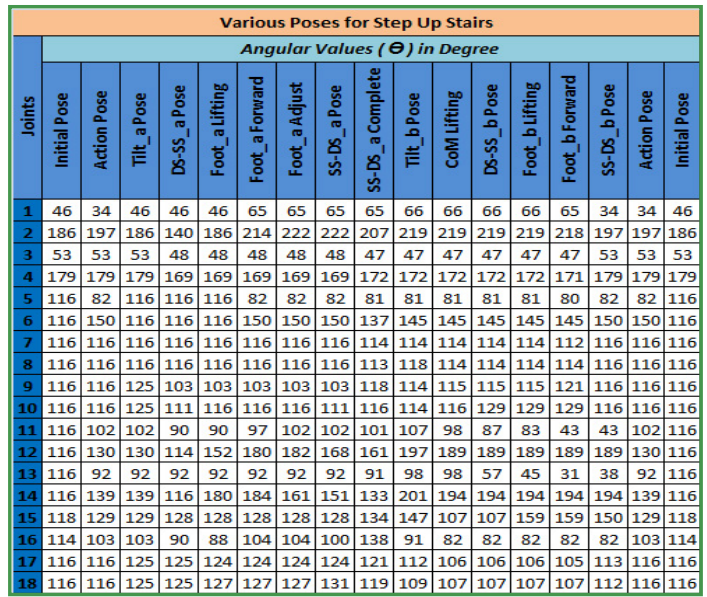

(a) Tabulated format of the Step Up constraints.

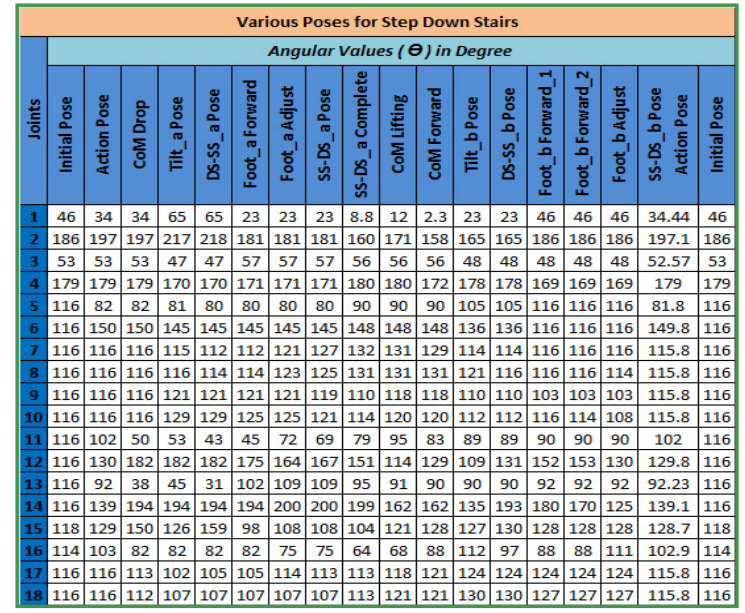

(b) Tabulated format of the Step Down constraints.

Figure 9. Tabulated values of the corresponding angular positions of the joint actuators for various poses of Ascending and Descending Steps

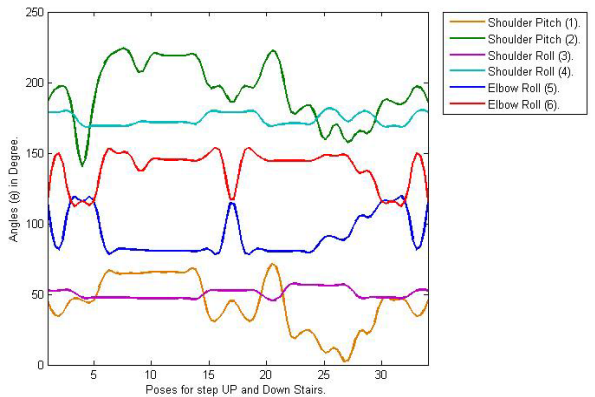

Figure 10. Upper torso mirror actuator movement patterns to execute the gaits for Step Up and Down Stairs

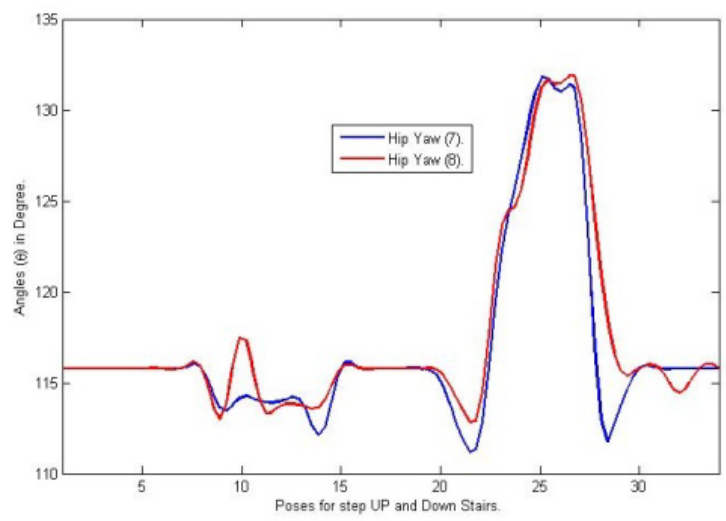

Figure 11. Motion trajectories of Hip Yaw mirror actuators while performing Up and Down Stairs

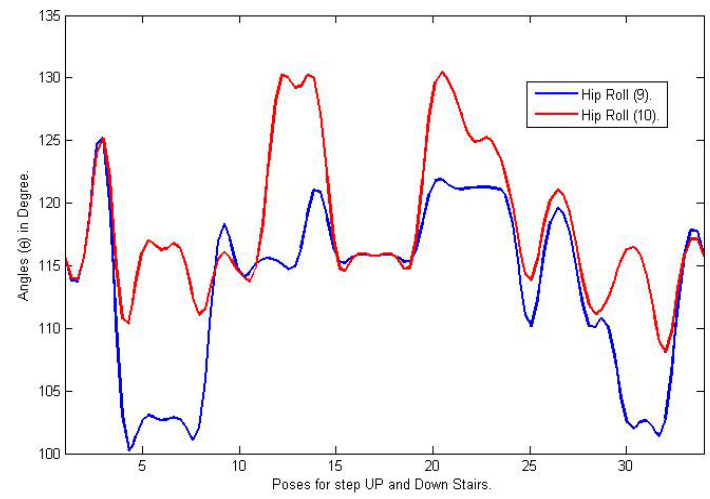

Figure 12. Motion trajectories of Hip Roll mirror actuators

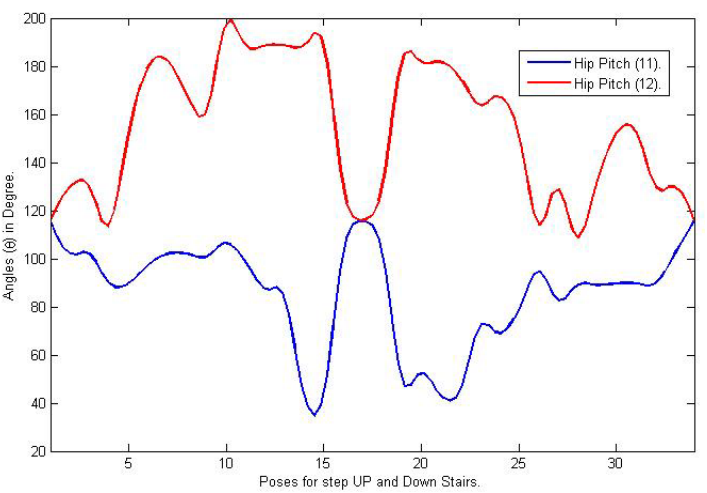

Figure 13. Motion trajectories of Hip Pitch mirror actuators

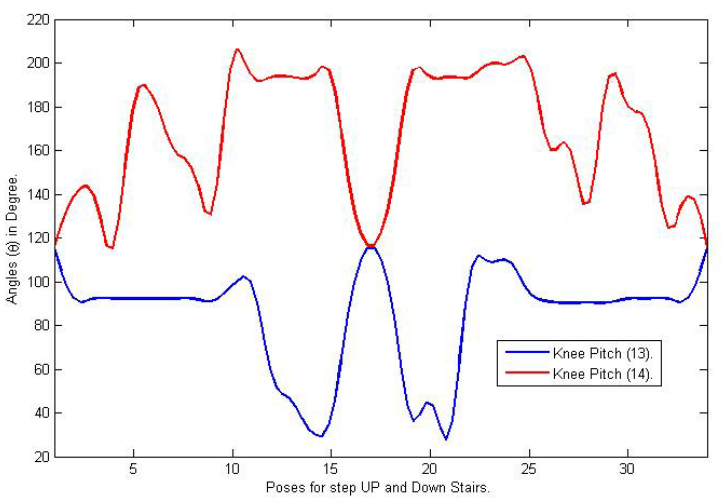

Figure 14. Motion trajectories of Knee Pitch mirror actuators 


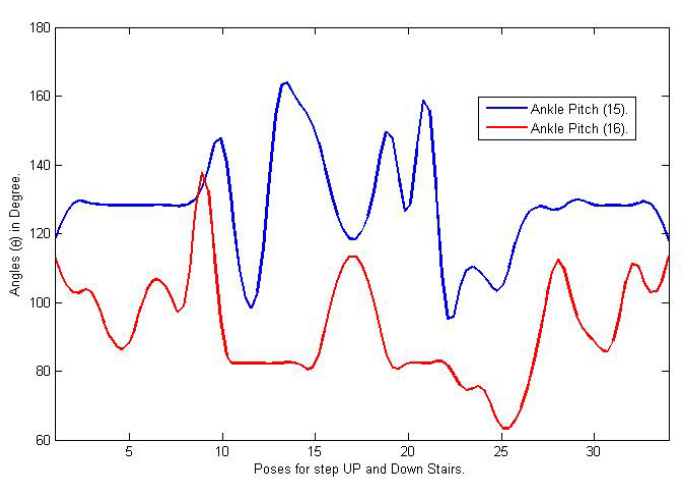

Figure 15. Motion trajectories of Ankle Pitch mirror actuators for Up and Down Steps of the Stairs

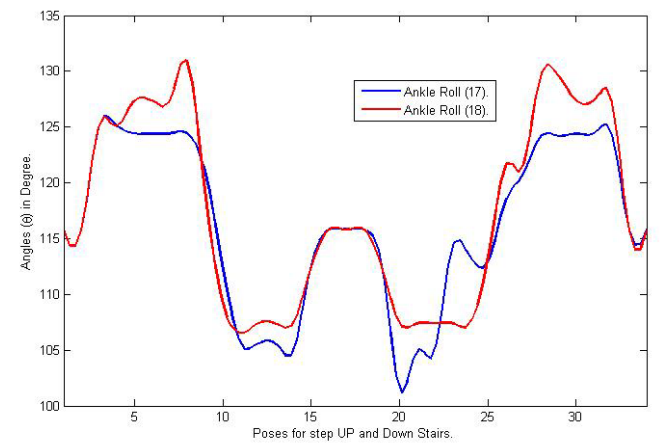

Figure 16. Actuator Motion trajectories for Ankle Roll mirror joints while stepping Up and Down Stairs

The behavior of the Hip Yaw and Ankle Roll joint actuators are showing almost same trajectory paths for their corresponding mirror actuators, indicating in Figure 11 and Figure 16. These characteristics also represents that the $\mathrm{z}$-axis assignment for these joint coordinates are in the same direction. Although the motion trajectories for Hip Roll mirror actuators show a little bit diversion, Figure 12, their $\mathrm{z}$-axis for local frames are also in the same direction. All the other Pitch actuators which are mounted at the lower torso of the robot present the opposite deflection characteristics for their corresponding mirror actuators because of the z-axis assignment of the joint frames are in opposite direction to each other. These characteristics are represented from Figure 13 to Figure 15. Basically these natures of the joint actuators are significant to perform the stable gait for ascending and descending steps of the stairs. The first half or the each graphs represents the pattern for stepping up stairs. The fashion of step down stairs can be expressed by the second half of the graphs, shown from Figure 10 to Figure 16.

\section{Conclusions}

As the experiment is a part of the main project to establish an optimal navigation system in indoor environment, the robustness of the designed gait is ensured through FK and IK analysis to identify the D-H parameters and imposing GAT or G-T formulation technique. The CoP-CoM tracking strategy is formulated based on the various patterns of the footsteps and zigzag motion of the CoP point where the CoM follows the CoP movement path. The ZMP concept is imposed to have the basic strategy of this analysis. As no sensors are used to acknowledge the environment or contact points on the ground, the CoP point is selected based on the percent of total pressure changes on the straight line between the ankle points of two legs. The percent of pressure is calculated automatically contingent upon a mathematical equation which is excogitated from the predefined step length, step angles and foot lengths. The robot performs its task to step over the stairs comparatively in slow motion but in a robust way by maintaining its torso in upright position. Basically a human like walking gait is tried to design that is experimented using the BIOLOID system and the joint movement behaviors of this robot are analyzed and presented in this paper. The experimental result shows that such kind of gait is feasible to implement on a real size humanoid robot to establish a navigation strategy in indoor environment.

\section{ACKNOWLEDGEMENTS}

The authors would like to thank their honorable parents. They also would like to express their gratitude to the Ministry of Higher Education (MOHE), Malaysia, in funding the project through the Fundamental Research Grant Scheme (FRGS).

\section{REFERENCES}

[1] J. Christensen, J. L. Nielsen, M. S. Svendsen and P. F. Orts, Development, Modeling and Control of a Humanoid Robot. Master's Thesis, Spring 2007. Department of Electronic Systems, Alborg University, Alborg, Denmark.

[2] C. Venkatayogi, Simulation of a Humanoid Robot. In partial fulfillment of the requirements for the degree Master of Science 2007, Rush College of Engineering and Technology of Ohio University, USA.

[3] M. R. A. Saavedra, Stable Locomotion of Humanoid Robots based on Mass Concentrated Model. Ph.D. Thesis, Department of System and Automation Engineering, Universidad Carlos III de Madrid, Madrid, Spain.

[4] C. Chevallereau, G. Bessonnet, G. Adda and Y. Aoustin, Bipedal Robots Modeling, Design and walking Synthesis (ISTE Ltd. and John Wiley \& Sons, Inc. 2009.)

[5] I. W. Park, J. Y. Kim, S. W. Park and J. H. Oh, Development of Humanoid Robot Platform KHR-2 (KAIST Humanoid Robot - 2). IEEE/RAS International Conference on Humanoid Robots, 2004. Vol. 1, 292-310.

[6] I. W. Park, J. Y. Kim, J. Lee and J. H. Oh, Mechanical Design of Humanoid Robot Platform KHR-3(KAIST Humanoid Robot - 3: HUBO). Proceedings of 2005 th IEEE/RAS International Conference on Humanoid Robots. 2005. 321-326.

[7] K. Kaneko, K. Miura, F. Kanehiro, M. Morisawa, S. Nakaoka 
and S. Kajita, Cybernetic Human HRP-4C. Proc. of 9th IEEE-RAS International Conference on Humanoid Robots, Paris, France, Dec. 7-10, 2009. 7-14.

[8] M. Akhratuzzaman and A. A. Shafie, Advancement of Android and Contribution of Various Countries in the Research and Development of the Humanoid Platform. International Journal of Robotics and Automation (IJRA), Volume (1): Issue (2), 2010. Computer Science Journal (CSC Journal), Malaysia. 43-57.

[9] M. Akhtaruzzaman and A. A. Shafie, Evolution of Humanoid Robot and Contribution of Various Countries in Advancing the Research and Development of the Platform. Proc. of International Conference on Control, Automation and Systems (ICCAS), KINTEX, Gyeonggi-do, Korea, Oct. 27-30, 2010. 1021-1028.

[10] C. Fu and K. Chen, Gait Synthesis and Sensory Control of Stair Climbing for a Humanoid Robot. IEEE Transactions On Industrial Electronics, Vol. 55, No. 5, May 2008, 2111-2120.

[11] S. H. Kim and Y. Sankai, Stair Climbing Task of Humanoid Robot by Phase Composition and Phase Sequence. 2005 IEEE International Workshop on Robots and Human Interactive Communication, ROMAN 2005. 531-536.

[12] T. H. S. Li, Y. T. Su, C. H. Kuo, C. Y. Chen, C. L. Hsu and M. F. Lu, Stair-Climbing Control of Humanoid Robot using Force and Accelerometer Sensors. SICE Annual Conference 2007 Sept. 17-20, 2007, Kagawa University, Japan. 2115-2120.

[13] P. Michel, J. Chestnutt, S. Kagami, K. Nishiwaki, J. Kuffner and T. Kanade, GPU-accelerated Real-Time 3D Tracking for Humanoid Locomotion and Stair Climbing. Proc. of the 2007 IEEE/RSJ International Conference on Intelligent Robots and Systems, San Diego, CA, USA, Oct 29 - Nov 2, 2007. 463-469.

[14] M. Akhtaruzzaman and A. A. Shafie, Geometrical Analysis on BIOLOID Humanoid System Standing on Single Leg. Proc. of 2011 4th International Conference on Mechatronics (ICOM `11), 17-19 May 2011, Kuala Lumpur, Malaysia.

[15] M. Akhtaruzzaman and A. A. Shafie, An Attempt to Develop a Biped Intelligent Machine BIM-UIA. Proc. of 2011 4th International Conference on Mechatronics (ICOM '11), 17-19 May 2011, Kuala Lumpur, Malaysia.

[16] M. Akhtaruzzaman and A. A. Shafie, A Novel Gait for Toddler Biped and its Control Using PIC 16F877A. Proc. of 2011 4th International Conference on Mechatronics (ICOM '11), 17-19 May 2011, Kuala Lumpur, Malaysia.

[17] M. A. Akhter. Accumulation of Research, LAP Lambert Academic Publishing, 2011.

[18] M. Eaton, Further Explorations in Evolutionary Humanoid Robotics. Proc. of 12th International Symposium on Artificial Life and Robotics (ISAROB), January $25-27,2007$, Oita, Japan. 133-137.

[19] F. A. Siddiky, N. H. Siddique and M. Tokhi, A New Approach to Design and Control of Biped Robot with Minimal Number of Actuators. Proc. of International Conference on Climbing and Walking Robots and Support Technologies for Mobile Machines, 09th September 2009. 115-122.

[20] A. Konno, K. Nagashima, R. Furukawa, K. Nishiwaki, T. Noda, M. Inaba and H. Inoue, Development of a Humanoid
Robot Saika. Proc. of IROS 1997. 805-810.

[21] D. Wollherr, M. Hardt, M. Buss and O. V. Stryk, Actuator Selection and Hardware Realizationof a Small and Fast-Moving, Autonomous Humanoid Robot. Proc. of the 2002 IEEE/RSJ International Conference on Intelligent Robots and Systems, Sept. 30 - Oct. 4, 2002, Lausanne, Switzerland. 2491-2496.

[22] E. Choong, C. M. Chew, A. N. Poo and G. S. Hong. Mechanical Design of an Anthropomorphic Bipedal Robot. First Humanoid, Nanotechnology, Information Technology, Communication and Control Environment and Management (HNICEM) International Conference, March 27-30, 2003, Manila, Philippines.

[23] R. Beira, M. Lopes, M. Praca, J. S. Victor, A. Bernardino, G. Mettay, F. Becchiz and R. Saltaren, Design of the Robot-Cub (iCub) Head. Proc. of IEEE International Conference on Robotics and Automation, May 2006, Orlando.

[24] D. Gouaillier, V. Hugel and P. Blazevic, Mechatronic design of NAO humanoid. Proc. of 2009 IEEE International Conference on Robotics and Automation, Kobe International Conference Center, Kobe, Japan, May 12-17, 2009. 769-774.

[25] V. V. Madadi and S. Tosunoglu, Design and Development of a Biped Robot. Proc. of the 2007 IEEE International Symposium on Computational Intelligence in Robotics and Automation. Jacksonville, FL, USA, June 20-23, 2007. 243-247.

[26] Y. Matsusaka. History and Current Researches on Building a Human Interface for Humanoid Robots. In Proceedings of ZiF Workshop 2006. 109-124.

[27] T. Sugihara and H. Kobayashi. A Handy Humanoid Robot Navigation by Non-interruptive Switching of Guided Point and Synergetic Points. Proc. of the 8th IEEE-RAS International Conference on Humanoid Robots, December 1-3, 2008, Daejeon, Korea.

[28] J. S. Gutmann, M. Fukuchi and M. Fujita. A Modular Architecture for Humanoid Robot Navigation. Proc. of 2005 5th IEEE-RAS International Conference on Humanoid Robots, 5th Dec. 2005, 26-31.

[29] N. T. Phuong, D. W. Kim, H. K. Kim and S. B. Kim. An Optimal Control Method for Biped Robot with Stable Walking Gait. Proc. of the 8th IEEE-RAS International Conference on Humanoid Robots, December 1-3, 2008, Daejeon, Korea, 211-218.

[30] C. L. Shih. Ascending and Descending Stairs for a Biped Robot. IEEE Transactions On Systems, Man and Cybernetics, Part A: Systems And Humans, Vol. 29, May 1999, 255-268.

[31] Y. Guan, K. Yokoi, N. E. San and K. Tanie. Feasibility of Humanoid Robots Stepping over Obstacles. Proceedings of 2004 IEEWRSJ International Conference on intelligent Robots and Systems. September 28 -October 2,2004, Sendal, Japan. 130-135.

[32] Y. Guan, N. E. Sian and K. Yokoi. Motion Planning for Humanoid Robots Stepping over Obstacles. IEEE/RSJ International Conference on Intelligent Robots and Systems, (IROS 2005), 2005. 363-369.

[33] G. Capi, Y. Nasu, L. Barolli and K. Mitobe. Real time gait generation for autonomous humanoid robots: A case study for walking. Robotics and Autonomous Systems 42 (2003), Elsevier Science B.V. 107-116. 
[34] J. Chestnutt, M. Lau, G. Cheung, J. Kuffner, J. Hodgins and T. Kanade. Footstep Planning for the Honda ASIMO Humanoid. Proceedings of the 2005 IEEE International Conference on Robotics and Automation, Barcelona, Spain, April 2005. 629-634.

[35] D. Katic and M. Vukobratovic. Survey of Intelligent Control Techniques for Humanoid Robots. Journal of Intelligent and Robotic Systems 37: 2003. Kluwer Academic Publishers. Printed in the Netherlands. 117-141.

[36] C. Chevallereau, G. Bessonnet, G. Adda and Y. Aoustin. Bipedal Robots Modeling, Design and walking Synthesis. ISTE Ltd. and John Wiley \& Sons, 2009. 47-70.

[37] K. Kaneko, F. Kanehiro, S. Kajita, K. Yokoyama, K. Akachi, T. Kawasaki, S. Ota and T. Isozumi. Design of Prototype Humanoid Robotics Platform for HRP. Proceedings of the 2002 IEEE/RSJ Intl. Conference on Intelligent Robots and Systems EPFL, Lausanne, Switzerland. October 2002. 2431-2436.

[38] D. Mewes and A. Heloir (2008). The Uncanny Valley. Seminar report, DFKI and Saarland University, 2008, [Online]. Available: http://embots.dfki.de/doc/seminar_ss09/writeup\%20uncanny $\% 20$ valley.pdf

[39] J. Carpenter, J. M. Davis, N. E. Stewart, T. R. Lee, J. D. Bransford and N. Vye. Gender Representation and Humanoid Robots Designed for Domestic Use. International Journal of Social Robots, March 19, 2009. Springer Science \& Business Media. 261-265.

[40] M. Akhtaruzzaman and A. A. Shafie. Evolution of Humanoid Robots and Contribution of Various Countries in Advancing the Research and Development of the Platform. The International Conference on Control, Automation and Systems (ICCAS), Oct. 27-30, 2010 in KINTEX, Gyeonggi-do, Korea. 1021-1028.

[41] M. Stilman, K. Nishiwaki, S. Kagami and J. J. Kuffner.
Planning and Executing Navigation Among Movable Obstacles. Proceedings of the 2006 IEEE/RSJ International Conference on Intelligent Robots and Systems, October 9 - 15, 2006, Beijing, China. 820-826.

[42] R. Simmons and S. Koenig. Probabilistic Robot Navigation in Partially Observable Environments. Proceedings of the International Joint Conference on Artificial Intelligence (IJCAI '95), July, 1995. 1080-1087.

[43] R. Tse, A. Tay and W. Hutama. Robot Navigation using KFLANN Place Field. Proceedings of the 2008 IEEE International Conference on Systems, Man and Cybernetics (SMC 2008), 12-15 Oct. 2008, Singapore. 3034-3039.

[44] M. Abdallah and A. Goswami. A Biomechanically Motivated Two-Phase Strategy for Biped Upright Balance Control. Proceedings of the 2005 IEEE International Conference on Robotics and Automation Barcelona, Spain, April 2005. 2008 -2013 .

[45] A. Goswami. Kinematic and dynamic analogies between planar biped robots and the reaction mass pendulum (RMP) model. 8th IEEE-RAS International Conference on Humanoid Robots. 1-3 Dec. 2008, Daejeon, Korea. $182-188$.

[46] C. Zhou, P. K. Yue, J. Ni1 and S. B. Chan. Dynamically Stable Gait Planning for a Humanoid Robot to Climb Sloping Surface. Proceedings of the 2004 IEEE Conference on Robotics, Automation and Mechatronics Singapore, 1-3 December, 2004. 341-346.

[47] P. Gibbons, M. Mason, A. Vicente, G. Bugmann and P. Culverhouse. Optimization of Dynamic Gait for Bipedal Robots. Proceedings of the 2009 IEEE-RAS Intl. Conf. On Humanoid Robots (Humanoids 2009), Paris(France), 2009, December 7-10. 9-14.

[48] T. Takenaka. The control system for the Honda Humanoid Robot. Published by Oxford University Press on behalf of the British Geriatrics Society. Age and Ageing 2006. 35-S2: ii24-ii26. 